\title{
LA UTILIZACIÓN DEL ESPACIO EN CONTEXTOS ICÓNICOS: EL CASO DE LOS NUMERALES EN LA LENGUA DE SIGNOS ESPAÑOLA
}

\author{
AnA Ma FERnÁndez Soneira \\ Universidade de Vigo \\ Grupo de investigación sobre lenguas signadas \\ anafe@uvigo.es
}

\begin{abstract}
Resumen
La explotación del espacio sígnico con fines gramaticales es una de las características más específicas de las lenguas de signos ${ }^{1}$. En este trabajo nos proponemos analizar el uso del espacio, en la lengua de signos española, unido a la expresión de información numérica en contextos con un alto grado de iconicidad. La expresión de magnitudes numéricas en la LSE es icónica. Los números del 1 al 10 se expresan con los diez dedos de las manos y a partir del 11 podemos signarlos representándolos en el espacio sígnico. Al lado de esta expresión base hay contextos en los que, bien dependiendo de la información que queramos transmitir bien del signo al que se una el numeral, nos encontramos con procedimientos de expresión más específicos y altamente icónicos, algunos de ellos ya gramaticalizados en la lengua y otros más discursivos. Intentaremos, en este trabajo, realizar una taxonomía de estos procedimientos y para ello analizaremos la explotación del espacio sígnico como si recrease un mapa temporal para la expresión de unidades de calendario, la explotación del espacio unida al uso de las manos como referentes icónicos para expresar información numérica asociada a valores de orden, localización e igualdad referencial y, por último nos referiremos a casos en los que la información numérica asociada a determinados conceptos se expresa a través de procedimientos altamente icónicos que se apoyan también en las posibilidades de explotación del espacio sígnico como representación a escala del mundo real..
\end{abstract}

PALABRAS CLAVE: lengua de signos española, iconicidad, numerales, explotación del espacio sígnico.

\begin{abstract}
The grammatically-targeted use of sign space constitutes one of the most specific features of Sign Languages. The aim of this article is to analyse, in Spanish Sign Language (LSE), the use of space linked to the expression of numeric information in contexts that display a high degree of iconicity. The expression of numeral information in LSE is purely iconic. Numbers from 1 to 10 are phrased with the fingers of both hands; from 11 onwards, we may sign them by their representation in the sign space. Apart from this basic way of expression, there are contexts in which, depending either on the information we want to convey or on the sign attached to the numeral, we are faced with more specific and highly iconic procedures, some of them already grammaticalised, some others more discursive. It is our aim to establish a taxonomy of such procedures by analysing: a) the use of sign space as a recreation of a time map to express calendar units, b) the joint use of space and both hands as iconic referents to express numeric information connected with rank, location and referential equation, and c) some instances in which numeric information associated to specific concepts is expressed by highly iconic procedures that rely on the possibilities sign space has to be used as a scale representation of the real world.
\end{abstract}

KEY WORDS: Spanish Sign Language, Iconicity, Numerals, Sign Space Use.

1 A lo largo de este trabajo se emplean indistintamente los términos lenguas de signos, lenguas signadas, signo y seña. Todos ellos están recogidos en la bibliografía de estas lenguas. La utilización de signo y seña presenta bastante controversia. La comunidad sorda prefiere el término signo en su gran mayoría, mientras que los investigadores suelen preferir el término seña, puesto que toda lengua natural es un sistema de signos por lo que la denominación lengua de signos es redundante (vid. Gascón, 1998). Las denominaciones seña y lengua de señas son además las empleadas por la comunidad hispanoamericana. 


\section{Introducción ${ }^{2}$}

La explotación del espacio sígnico con fines gramaticales es una de las características más específicas de las lenguas de signos. En este trabajo nos proponemos analizar el uso del espacio, en la lengua de signos española (en adelante LSE), unido a la expresión de información numérica en contextos con un alto grado de iconicidad.

El apartado 2 explica la metodología que hemos adoptado para elaborar los ejemplos que nos han ayudado a describir la expresión icónica de los numerales. En los apartados 3 y 4 se exponen unas breves nociones sobre la iconicidad -en las lenguas orales y en las signadasy el espacio. En el apartado 5 se describen, en primer lugar, las formas de expresión básica de los numerales en la LSE para, a continuación, abordar la expresión de los numerales en contextos con un alto grado de iconicidad. Por último, en el apartado 6, presentaremos unas breves reflexiones a todo el trabajo.

\section{Metodología}

Para la ejemplificación de los contenidos teóricos nos valdremos de datos que hemos obtenido a través de diferentes procedimientos. En primer lugar creamos un texto en el que se hablaba de situaciones en las que aparecía información numérica relacionada con los contextos que queríamos describir. Este texto fue interpretado por un colaborador sordo y grabado, posteriormente, en vídeo ${ }^{3}$. Los contenidos de esta grabación, cuyo texto original presentamos a continuación, fueron revisados también con otros colaboradores de nuestro proyecto de investigación ${ }^{4}$.

Las navidades pasadas me tocó la lotería. Cuando vi en la tele que los cinco números coincidían con mi boleto, no me lo podía creer. Otras veces había tenido la terminación e incluso una vez las tres últimas cifras, pero nunca el premio gordo.

Ahora mi vida ha cambiado un poco. Lo primero que hice fue comprarme un buen coche, con seis marchas. Además cada quince días hago un pequeño viaje de fin de semana. Los otros dos fines de semana me quedo para ir a ver jugar a mi equipo de fútbol. Ahora vamos cuartos en la clasificación pero si ganamos esta semana nos pondremos segundos. También voy al estadio los miércoles cuando toca partido europeo. Hemos jugado los cuartos de final, ganamos y ahora estamos en semifinales. ¡Ojalá lleguemos a la final!

El dinero de la lotería también me servirá para pagar mi boda. Me caso la segunda semana de julio. Mi vida ahora es más relajada.

En segundo lugar, nos hemos servido de otros materiales del corpus perteneciente al grupo de investigación sobre lenguas signadas de la Universidad de $\mathrm{Vigo}^{5}$; hemos revisado, por

2 Quiero agradecer los comentarios y sugerencias aportadas por José María García-Miguel, Inmaculada Báez, Carmen Cabeza y Jorge L. Bueno, a quien especialmente le agradezco que llevase a cabo la versión en inglés del resumen de este artículo.

3 Este vídeo fue mostrado en la presentación de la ponencia que ha dado origen a este trabajo, en el segundo congreso internacional de Aflico, celebrado en Lille (10-12 de mayo de 2007).

4 «Bases para el análisis lingüístico de la lengua de signos española» (Referencia: HUM2006-10870/FILO), proyecto concedido por el Ministerio de Educación y Ciencia.

5 http://webs.uvigo.es/lenguadesignos/sordos/home 
una parte, cuestionarios con los que tratamos de obtener información sobre las expresiones de cantidad existentes en la lengua de signos española (LSE) y el uso de expresiones temporales $\mathrm{y}$, por otra parte, dos conversaciones semi-dirigidas: en la primera dos informantes hablaban de temas relacionados con el paso del tiempo; en la segunda, nuevamente dos informantes conversaban sobre temas que exigían un uso abundante de expresiones de cantidad: la familia, los amigos, los estudios, el trabajo, los recuerdos del pasado, etc.

Todas las producciones se grabaron en vídeo. Una vez realizada la grabación, revisamos las imágenes y glosamos las producciones. Para realizar el presente estudio hemos contado con la ayuda (directa) de tres informantes, dos sordos y un intérprete, signantes de LSE (variedad de Galicia) y con las producciones de otros informantes grabadas en vídeo ${ }^{6}$.

Por último hemos revisado diversos materiales del LSE, tales como algunos vídeos del método didáctico Signar -editado por la Confederación Estatal de Sordos Españoles-y otros materiales divulgativos, que nos han servido para corroborar los contenidos teóricos que presentaremos en las siguientes líneas.

Estas muestras presentan, en términos generales, un carácter más formal, por tratarse bien de contenidos didácticos, bien de contenidos divulgativos y/o institucionales. Las muestras mencionadas en líneas anteriores fueron registradas en un contexto más informal.

La transcripción de los ejemplos utilizados, tanto los cuestionarios como la conversación, se ha hecho empleando un sistema de glosas basado en propuestas de Klima \& Bellugi (1979) y Johnston (1996), adaptado a la lengua de signos española.

\section{La iconicidad}

En este trabajo analizamos la iconicidad asumiendo como punto de partida la división tradicional del signo, propuesta por Peirce (1902), quien establecía tres tipos de signos: índices, iconos y símbolos. Dentro de los signos icónicos, es decir, aquellos que pueden representar un referente por similitud, distinguía entre imágenes, diagramas y metáforas.

Un icono es un Representamen cuya Cualidad Representativa es una Primeridad de él como un Primero [...] Los que participan de cualidades simples o Primera Primaridad son imágenes; los que representan las relaciones, principalmente diádicas o consideradas tales, de las partes de una cosa mediante relaciones análogas en sus propias partes, son diagramas; los que representan el carácter representativo de un representamen mediante la representación de un paralelismo en alguna otra cosa son metáforas (Peirce, 1987: 262-263).

Basándonos en esta clasificación podemos hablar de tres tipos de iconicidad: imagética, diagramático y metafórica. Siguiendo las ideas de Peirce, destacan los trabajos de Jakobson (1971) y Haiman (1980) quienes defienden que hay muchas características en las lenguas que no son arbitrarias. Jakobson concibe la lengua como un instrumento para una finalidad y establece una serie de funciones del lenguaje relacionadas con el acto comunicativo (expresiva, apelativa, poética, referencial, fática y metalingüística); más adelante hablaremos de

6 Quiero agradecer la ayuda inestimable de Juan Ramón Valiño, Araceli Santos y Francisco Eijo. Este último ha sido, además de informante, protagonista del vídeo mencionado con anterioridad y ha colaborado en el montaje final de las fotos que aparecen a lo largo del trabajo. 
la referencial, la que se relaciona con el contexto del acto comunicativo. En su obra sobre la esencia del lenguaje, Jakobson resumía de la siguiente manera la concepción de Peirce:

Peirce likewise makes a clear-cut distinction between the «material qualities», the signans of any sign, and its «immediate interpretant», that is, the signatum. Signs [...] offer three basic varieties os semiosis, three distinct «representative qualities» based on different relationships between the signans and signatum (Jakobson 1971: 346-347).

En la iconicidad imagética la relación de semejanza que se establece entre el signo y su referente, en las lenguas orales, se restringe, generalmente, al sonido de dicho referente en casos como las onomatopeyas.

En la iconicidad diagramática un enunciado y su referente comparten el orden o el esquema de sus componentes. Haiman (1980) señala dos tipos, con la intención de remarcar que la estructura lingüística no es independiente del significado.

Although linguistic signs in isolation are simbolic, the system or grammar which relates them may be diagrammatically iconic in two ways: (a) by isomorphism, a biunique correspondence tends to established between signans and signatum; (b) by motivation, the structure of language directly reflects some aspect of the structure of reality (Haiman, 1980: $515)$.

Este tipo de iconicidad se enmarca -en las lenguas orales- principalmente en el dominio de la sintaxis, aspecto en el que se ha incidido en estudios recientes (cfr. Haiman 1985; Simone 1995). Encontramos casos de motivación icónica en el orden de los constituyentes dentro de la secuencia-la proximidad formal refleja una mayor ligazón conceptual (García-Miguel 1996: 420) -, en la colocación de los morfemas dentro de la palabra, etc.

La iconicidad metafórica hace referencia a contextos en los que se establece una relación entre el significado de un signo (lingüístico) y algo diferente del mismo.

Frente a la visión arbitraria del lenguaje adoptada a partir de las ideas de Saussure, lo que la lingüística actual defiende es que la estructura del lenguaje y la de las lenguas refleja nuestra percepción del mundo extralingüístico, tal y como expone García-Miguel (1996: 422):

[...] toda a estrutura lingüística está motivada finalísticamente polas condicións de uso das lenguas nas situacións comunicativas e polas capacidades cognitivas dos seres humanos. A diversidade das linguas é unha proba evidente de arbitrariedade relativa; a existencia de universais é proba de que as linguas non poden variar arbitrária e ilimitadamente. Unha das tarefas mais interesantes da lingüística actual é a procura de motivacións para os universais lingüísticos.

En cuanto a la iconicidad en las lenguas de signos, el tratamiento de esta noción se ha realizado desde diferentes posturas: hay lingüistas que no tratan como pertinente este concepto e incluso han tratado de reducir su importancia para poder estudiar las lenguas de signos con parámetros estructuralistas (por ejemplo, Frishberg 1975 o Armstrong 1983); otros creen que su tratamiento nos lleva a una visión limitada sobre el lenguaje y las lenguas. Es evidente que, desde un punto de vista teórico, la iconicidad adquiere un sentido pleno a nivel de la función referencial. 
Los trabajos de Cuxac (1996, 2000) para la lengua de signos francesa (LSF) han relanzado el estudio de esta noción y han servido para establecer un nuevo modelo de análisis. Este autor parte de la iconicidad como base descriptiva de las lenguas de signos. Es evidente, debido a su carácter viso-gestual, que la iconicidad imagética es la más común en las lenguas signadas. Ya hemos mencionado que este tipo de iconicidad hace referencia a la similitud que se establece entre un signo y el objeto al que se refiere.

Cuxac parte de la idea de que la construcción de referencias actanciales y espaciales específicas es similar en todas las lenguas de signos; considera que es posible el establecimiento de una comunicación icónica a través de estructuras mínimas de gran iconicidad. Agrupa estas estructuras bajo el término transferts (transferencias): «il s'agit d'operations qui permettent, en amont, de transférer, en les anamorphosant faiblement, des expériences réelles ou imaginaires dans l'univers discursif tridimensionnel apellé «espace de signation» (Cuxac 2000: 24).

Así pues, esta operación permite transferir el mundo real en el discurso signado cuatridimensional (las tres dimensiones del espacio y la dimensión del tiempo). En este tipo de estructuras discursivas la iconicidad imagética juega un papel muy importante, y está menos presente en los signos estandarizados, es decir, en los signos convencionales (símbolos).

Sallandre (2006) afirma, en su estudio sobre el espacio y la iconicidad en la LSF, que la gran iconicidad tiene una mayor presencia en contextos discursivos que en contextos explicativos, afirmación que nos parece aplicable a la LSE. Esta afirmación se justifica en el hecho de que las lenguas de signos ofrecen la posibilidad de expresar una misma situación de dos maneras diferentes, bien con signos estándar bien a través de estructuras más icónicas que nos permiten describir una acción valiéndonos del espacio sígnico para asumir diferentes roles simultáneamente, describir formas, colocar elementos en diferentes puntos espaciales, etc.; este tipo de recursos se utilizan normalmente cuando contamos una historia, hablamos de algo que nos ha pasado,... y suelen estar ausentes en contextos más formales.

En cuanto a las estructuras estándar, Cuxac parte de la premisa de que en estas la iconicidad no puede deducirse de la intención del locutor de emitir sus mensajes haciéndolos similares a las experiencias que él transmite. Se centra en cuatro grandes bases estructurales: 1) la organización del léxico y el carácter molecular de los signos estándar, 2) un orden preferente de los elementos en la cadena «orden de la iconicidad», 3) la multilinearidad paramétrica de las informaciones semántico-sintácticas y 4) la utilización pertinente del espacio para marcar las relaciones semánticas.

Cuando nos referimos al léxico estándar de las lenguas signadas nos encontramos con una iconicidad reducida o desfigurada. Cuando abordamos el estudio de las relaciones sintácticas y semánticas adquiere valor el uso del espacio «iconizado diagramáticamente».

La mayoría de los estudios realizados sobre la iconicidad en las lenguas de signos se han centrado en la iconicidad imagética, si bien en los últimos años se ha profundizado también en la diagramática para el análisis de las referencias de persona, espacio y tiempo (Cuxac 2003, Emmorey 2002, Sallandre 2003 y 2006). Sallandre (2006: 240) observa que, en LSF, la presencia de la iconicidad diagramática se caracteriza por los señalamientos y la realización de signos estandarizados en el espacio sígnico concebido como un diagrama ${ }^{7}$ :

7 Equipara esta noción con la de Token Space establecida por Liddell (1995: 33): «The space that tokens inhabit is limited to the size of the physical space ahead of the signer in which the hands may be located while signing. I refer to a mental space grounded in all or part of the physical signing space as a Token Space». 
«Diagrammatic iconicity is a type of syntactical iconicity that is present in sign languages in the constructions of space, time and person references, as well as in the order of sign in the utterance».

Al igual que en las lenguas orales, la iconicidad diagramática estaría presente en el orden de los constituyentes dentro de la secuencia y, específicamente, en la expresión del espacio y del tiempo. En palabras de Sallandre (2006: 249):

[...] the essence of a diagram is that the relationships between its parts resemble the relationship between the parts of the object that it represents. [...] Generally, diagrammatic iconicity is visible in two main domains of natural languages: word order in the syntagmatic chain (in both spoken and signed languages) and space conceived of as a diagram [...] which he/she will refer to throughout discourse (especially in signed languages).

También en los últimos años han proliferado los estudios que abordan el análisis de las metáforas en las lenguas de signos (Wilcox 2000, Taub 2001). La iconicidad metafórica, aquella en la que los signos se usan en lugar de los objetos reales y el contexto de signación reproduce el contexto real al que se refiere, está muy presente en las lenguas de signos, sobre todo en el discurso. La utilización del espacio sígnico real, sin necesidad de acudir a un espacio imaginario, permite la posibilidad de expresar una metáfora de manera mucho más directa que en las lenguas orales, puesto que la información que queremos transmitir se copia en el espacio de la signación y se trata como si fuese la realidad misma.

\section{El espacio sígnico}

En las lenguas de signos, caracterizadas como lenguas viso-espacio-gestuales, el espacio juega un papel fundamental no solo en la articulación de los signos (como parámetro formativo) sino en la construcción de referencias temporales, espaciales y actanciales. Se trata, en palabras de Cuxac (2000: 261), de «une exploitation d'une ou plusiers dimensions de l'espace de signification afin de génerer des effects de sens».

Con el término espacio sígnico nos referimos al área en la que el signante localiza la totalidad de las señas que realiza. En él se reconocen varios puntos de articulación que pueden estar en el cuerpo o en el espacio alrededor del signante. Liddell (1990) estudió cómo se usaba la localización en ASL e introdujo el término locus para denominarla; distinguió además cuatro funciones que éste cumplía en distintos contextos sígnicos: la función articulatoria -presente en la realización de todos los signos-, la función tridimensional, propia de los verbos espaciales -el espacio se usa fundamentalmente para situar objetos tridimensionales-, una función deíctica localizadora (con la que se alude a la capacidad localizadora a través de la deixis), y una función de igualdad referencial que nos permite identificar unas determinadas localizaciones con unas determinadas entidades, como sucede en los verbos direccionales.

Las lenguas de signos permiten pues la explotación del espacio de formas específicas para expresar nociones gramaticales relacionadas con determinados signos. Los siguientes comentarios de Liddell (1995: 19) para la lengua de signos americana podrían aplicarse a diferentes lenguas de signos: «ASL allow signers to produce signs at, or direct signs toward, different locations within the signing space to indicate specific referents or to show specific locations. Pronouns, indicating verbs, locative verbs and classifier predicates, all have these capability». 
En este mismo estudio, Liddell, desde una perspectiva cognitiva, establece tres tipos de espacios mentales que aparecen en el discurso signado: Real Space, Token Space y Surrogate Space. Las funciones que mencionamos con anterioridad se enmarcarían dentro de esta nueva concepción del espacio. Se trata de tres posibilidades de explotación del espacio: en un espacio real localizamos y dirigimos los signos hacia posiciones reales y visibles (función deíctica localizadora), mientras que en los otros dos tipos son invisibles. El uso del espacio sustituto (surrogate) es propio de los contextos en los que se produce un cambio de roles mientras que en el espacio simbólico (token) los referentes se identifican por un clasificador o una seña dirigida hacia un punto del espacio (Liddell 1995: 28, 35):

....surrogates have the propierties of being invisible, being normal sized, having body features, being viewed as present with the signer, and having the capability of assuming either second person or third person roles in the discourse [...] Token space is a grounded mental space containing invisible entities that signs can be directed toward and that signers use for the purposes of communicating about events.

Sallandre (2006), tal y como hemos mencionado en líneas anteriores, equiparada esta noción con la de explotación diagramática del espacio sígnico; es decir, el signante crea un espacio concebido como un diagrama, en el que localiza los signos de su acto comunicativo. En él no solo aparecen las manos como articuladores sino que las propias manos así como otras partes del cuerpo pueden representar entidades estructurales (por ejemplo, en la figura 10 veremos cómo el cuerpo se convierte en un límite espacial); incluso la configuración puede representar más que una forma, puede representar una trayectoria, una localización...

La explotación del espacio como mapa a escala del mundo real nos permite representar, en la signación, el mundo extralingüístico. En este sentido, la noción del espacio no puede separarse de la de iconicidad, tal y como afirma Talmy (2006: 207):

In signed language, spatial structure is mainly represented by the «classificatory» subsystem, which systematically differs from the spoken language system. It marks finer spatial distinctions with its inventory of more structural elements, more categories, and more elements per category. It represents these distinctions independently in the expression, not bundled together into prepackaged schemas. And its spatial representations are largely iconic with visible spatial characteristics.

En este trabajo nos centraremos en la expresión de información numérica en un espacio simbólico e icónico. La articulación de los signos numerales se realiza en el espacio sígnico neutro, es decir, sin contacto con el cuerpo del signante; aquel adquiere otros valores en contextos espaciales en los que los signos no solo expresan una información numérica sino que además nos aportan una información de orden, de localización, etc. La explotación del espacio y las características del subsistema de clasificación de las lenguas de signos nos permiten representar muchos más elementos y categorías distintas juntas en una sola expresión (Talmy 2006: 236). Se trata de combinar conceptos individuales bajo ciertas limitaciones para representar un complejo conceptual. 


\section{La expresión de la cuantificación numérica en la LSE}

La cuantificación presenta en la LSE una gran variedad de formas de expresión ${ }^{8}$. En este artículo nos centramos en la expresión de la cuantificación definida, es decir, aquella que expresa un número exacto de referentes.

\subsection{Los numerales en la LSE}

La expresión de magnitudes numéricas en la LSE es icónica (las configuraciones de los signos numerales remiten a formas referenciales específicas). La LSE presenta un sistema decimal, es decir, diez signos básicos para los números cardinales que se pueden contar con los dedos. Los números del 1 al 10 se expresan con los diez dedos de las manos, extendiéndolos hacia arriba, empezando por el índice (de la mano dominante). A partir del 11 podemos signarlos representándolos en el espacio sígnico, reproduciendo su escritura, moviendo las configuraciones de izquierda a derecha, por ejemplo: 1-2 doce o con configuraciones especiales.

En LSE existen también diez signos básicos para los números ordinales del uno al diez que presentan la misma configuración que los cardinales pero se articulan con un giro de muñeca (la orientación final de la palma de la mano hacia el emisor) que les otorga un valor ordinal. A partir del once suele usarse un numeral cardinal unido al signo nominal.

Los numerales, por norma general, se posponen al nombre al que cuantifican. Sin embargo, la influencia del español hace que se produzcan interferencias, en este caso de tipo sintáctico, y que se anteponga en ocasiones el numeral en contextos no marcados. En los contextos marcados, por ejemplo para enfatizar una información cuantitativa, se antepone el numeral. Herrero Blanco (2009: 157) afirma que los numerales se anteponen a los nombres cuantificados de medida, peso, moneda, etc., señalando en primer lugar la cantidad a la que nos referimos y luego el referente.

Al lado de la expresión base de los numerales encontramos algunos recursos, propios de las lenguas de signos, que afectan también a estos cuantificadores, tales como la incorporación numeral unida a las expresiones temporales para expresar una referencia más específica o a los llamados tradicionalmente clasificadores de entidad para representar un número específico de entidades. Estos son procedimientos completamente gramaticalizados en la LSE.

Nuestra intención es centrarnos en contextos en los que, bien dependiendo de la información que queramos transmitir bien del signo al que se una el numeral, nos encontramos con procedimientos de expresión más específicos y altamente icónicos, algunos de ellos ya estandarizados en la lengua y otros más discursivos, relacionados en su mayoría con la expresión de referencias temporales y espaciales. A ellos dedicaremos el apartado siguiente.

8 En Fernández Soneira 2008 analizo los procedimientos existentes para la expresión de la cuantificación en la LSE. A él me remito para la ampliación de estos conceptos. 


\subsection{Los numerales en contextos icónicos}

Partiremos, en este apartado, de lo más estandarizado a lo más discursivo. En todos los casos el espacio se revela como el recurso que permite transferir el mundo real dentro del discurso signado.

\subsubsection{La explotación del espacio sígnico como si recrease un mapa temporal para} la expresión de unidades de calendario (sobre todo en contextos distributivos)

El uso de signos que marcan la alternancia y su desplazamiento en los diferentes planos espaciales permite reflejar la distribución del tiempo y su localización en un calendario. El espacio permite representar un mapa temporal.

(1) Cada quince días hago un viaje de fin de semana.

YO [MNODOM-HORIZ]CUATRO_SEMANAS[V,X,Y,Z]/PRIMERA[V]_TERCERA[Y] ${ }^{9}$

QUINCE DÍA[N:REP] CADA_DOS_SEMANAS[LOC X,Y,Z]

$$
\text { días sí/no, sí/no }
$$

VIAJE[N:REP][LOC X, Y, Z] FIN-DE-SEMANA

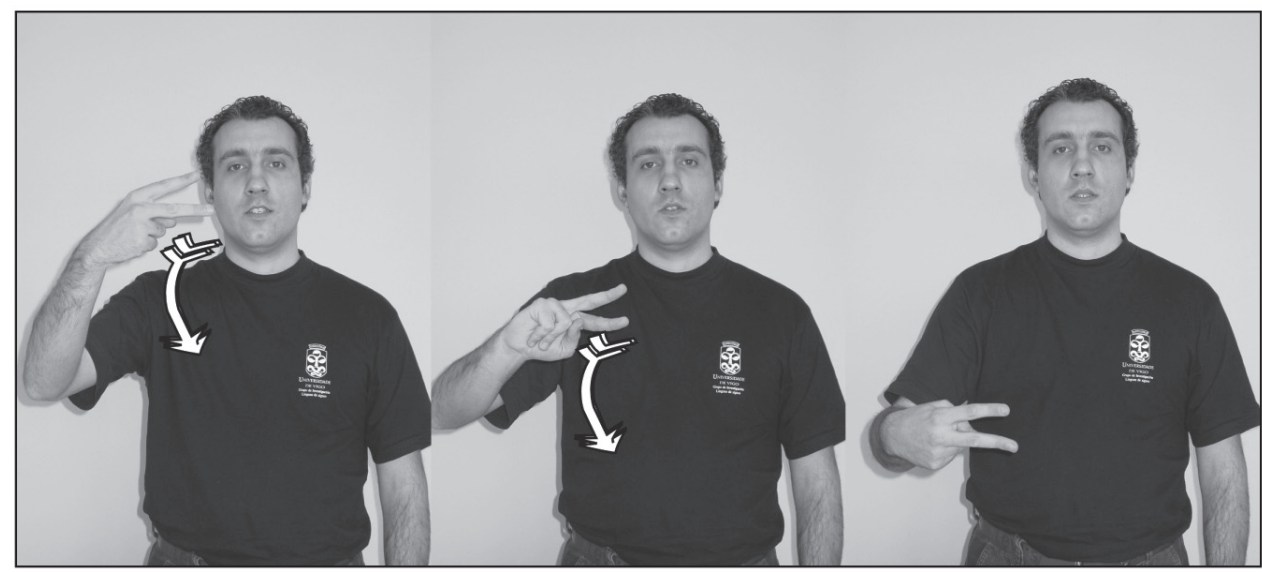

Figura 1.CADA_DOS_SEMANAS

En un primer momento el signante establece las referencias temporales en la mano pasiva (aspecto que tratamos en líneas posteriores) para marcar las semanas que se va de viaje, la primera y la tercera y después traslada las referencias al espacio sígnico neutro, tal y como muestra la figura 1, para indicar la frecuencia quincenal de sus viajes.

9 La glosa / indica que se trata de signos simultáneos. El signante mantiene la configuración CUATRO_SEMANAS en la mano izquierda, mano pasiva, mientras apoya los dedos índice y corazón de la mano dominante sobre los dedos índice y anular de la mano pasiva, los cuales representan la primera y la tercera semana del mes. 
(2) Deberías ir al gimnasio cada dos días, lunes, miércoles y viernes.

(TÚ) LUNES[LOC X] MIÉRCOLES[LOC Y] VIERNES[LOC Z] 2_[MONOM]DÍAS

CADA_DOS_DÍAS[LOC X, Y, Z] IR GIMNASIO ALGO

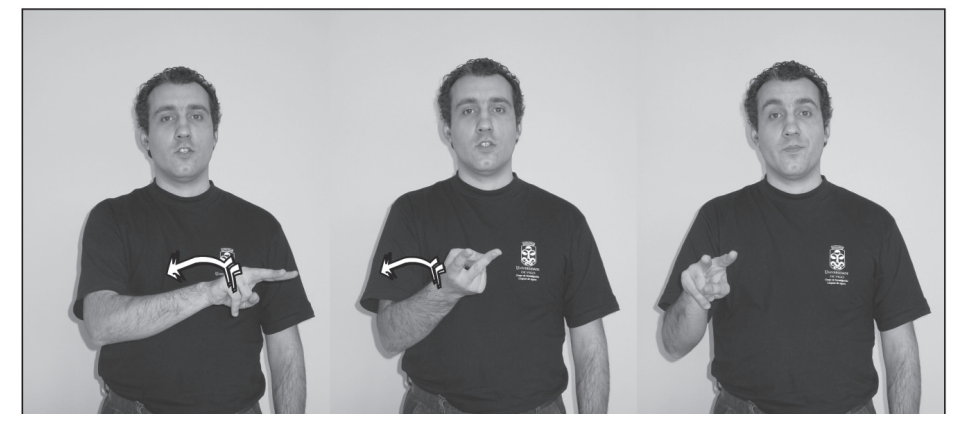

Figura 2. CADA_DOS_DÍAS

En ambos casos el signante elabora unas referencias temporales tomando el espacio como si fuese un calendario y, por tanto, la información referida a las semanas se articula desplazando el signo CADA_DOS_SEMANAS de arriba abajo, tal y como se suelen disponer estas en una representación de un mes, mientras que la información referida a los días de la semana se desplaza en el eje horizontal. Se crean puntos de referencia en el espacio. En ambos casos se usa el mismo signo, que tiene una articulación motivada, los dedos índice y corazón estirados, con la misma configuración que el numeral «2»y se mueve, como hemos dicho, en dos planos diferentes dependiendo de la información que queramos dar.

En el segundo de los ejemplos, se activa una porción del espacio en la que se localizan los signos que tienen valor temporal (LUNES[LOC X] MIÉRCOLES[LOC Y] VIERNES[LOC Z]) y en los mismos puntos espaciales se realiza a continuación el signo CADA_DOS_DÍAS. En estos contextos adquiere un valor esencial la mirada que actúa como activadora del espacio (contribuye a establecer puntos de articulación y de cambio de cuadro de referencia discursiva).

La expresión de referencias temporales puede realizarse, en LSE, a través de otro procedimiento icónico, completamente estandarizado en la LSE (su uso es sistemático y no depende completamente del contexto discursivo); consiste en el establecimiento de puntos de referencia en la mano pasiva. Esta se usa a modo de localizador identificándola nuevamente con un calendario, atribuyendo a los dedos una función de igualdad referencial, relevante cuando existe una relación entre un lugar y un referente. Se identifican unas determinadas localizaciones con unas determinadas entidades. En este caso, cada dedo de la mano se identifica con una semana del mes, con un orden establecido (el índice la primera y así sucesivamente hasta completar cuatro semanas). ${ }^{10}$

10 Liddell (2003: 223) se refiere a estas construcciones con el nombre de buoys: «Signers frequently produce signs with the weak hand that are held in a stationary configuration as the strong hand continues producing signs. Semantically they help guide the discourse by serving as conceptual landmarks as the discourse continues». 
(3) Me caso la segunda semana de julio.

JULIO [MNODOM-HORIZ]CUATRO_SEMANAS[V,X,Y,Z]/SEGUNDA[X] (YO) CASAR

Al igual que en el ejemplo 1, la mano pasiva adopta la configuración «4» (con los dedos orientados hacia la derecha del signante) y simultáneamente con el dedo índice de la mano dominante toca la punta del dedo corazón de la mano pasiva, el que representa la segunda semana del mes. En el ejemplo 1 el signante utilizaba los dedos índice y corazón de la mano dominante para tocar los dedos índice y anular de la pasiva (dejando el corazón en medio) para referirse a las semanas primera y tercera del mes.

Esta construcción permite no solo identificar una semana particular sino también expresar otros significados como «a lo largo de la semana» a través del movimiento del dedo índice por toda la extensión del dedo que se identifica con la semana de la que hablamos.

Este procedimiento aparece asociado no solo a referencias temporales sino a otros conceptos a los que, en un contexto determinado, se les atribuyen valores de orden. Es el caso de las relaciones familiares en LSE. Normalmente cuando hablamos del número de hermanos que tenemos y de nuestra posición, atendiendo a criterios de edad, utilizamos también la mano pasiva. En este caso, cada dedo representaría una entidad, un hermano.

$\mathrm{Al}$ igual que en el ejemplo anterior, hay un orden inherente que se repite en diferentes lenguas de signos. Hemos constatado, por ejemplo, que tanto en ASL (Liddell 2003: 325) o en LSF (Cuxac 2000: 242) como en LSE el dedo pulgar representa siempre el primer referente, el índice el segundo y así sucesivamente. El lugar remite a la entidad de la que estamos hablando. En LSE, si hablamos de cuatro unidades empleamos todos los dedos de la mano dominante menos el pulgar que se añade si hablamos de cinco o más entidades.

En estas construcciones la mano pasiva mantiene un signo estático, mientras que la dominante realiza los signos estándares. Cuxac (2000: 241) expresa de la siguiente manera el significado de esta construcción:

Mais la main arrêtée joue là le role d'un indice de permanence thématique ou syntagmatique et le récepteur du message ne peu faire autrement qu'appréhender visuellement cet indice en même temps que les signes standars émis par l'autre main se succédent, conférant à ceux-ci un indiscutable etiquetaje structural simultané.

En nuestra opinión este tipo de expresión se englobaría dentro de la iconicidad diagramática. El signante construye en el espacio (o en la mano pasiva) un calendario en el que articula los signos estándares. El espacio no se convierte en una representación a escala del mundo real sino en una interpretación por medio de un diagrama ${ }^{11}$. Este tipo de construcciones presentan, según Cuxac (apud Sallandre 2006: 252), dos características lingüísticas: «pointing which marks the continuation of time and space references and frozen signs inside a diagram».

11 En su trabajo sobre la iconicidad y el espacio en la LSF, Sallandre (2006: 250-251) ofrece un ejemplo de iconicidad diagramática a propósito de una secuencia temporal: el signante construye en el espacio una parrilla horaria con dos ejes: los días de la semana y las partes del día. En un determinado momento de la signación articula un signo estándar en dos referencias espaciales diferentes que reconocemos como momentos temporales gracias a la interpretación del espacio que construye al principio de la secuencia. 


\subsubsection{La explotación del espacio para expresar información numérica asociada a valores locativos}

En estos contextos nos encontramos ante un mapa espacial en el que podemos localizar entidades, asociarles un valor numérico, un orden, etc. Vamos a utilizar, para ejemplificar esta idea, contextos en los que se transmite la misma información de cantidad; empleamos la misma proforma (configuración «4»).

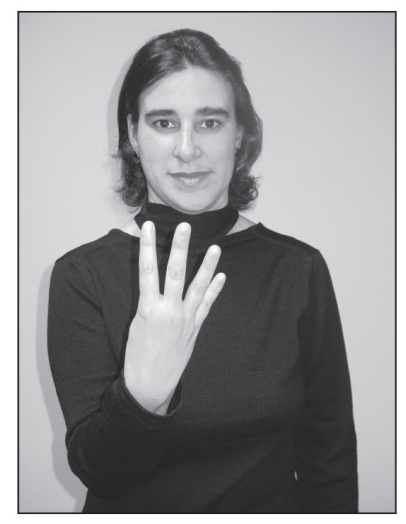

Figura 3. CUATRO

A través de ellos podremos ver como la posición de la mano en el espacio, la orientación de la palma de la mano, etc. nos permiten transmitir información diferente asociada a un mismo valor numérico.

(4) El Celta ocupa, en estos momentos, el cuarto puesto en la clasificación liguera. AHORA SIGNO[CELTA] CUARTO_PUESTO

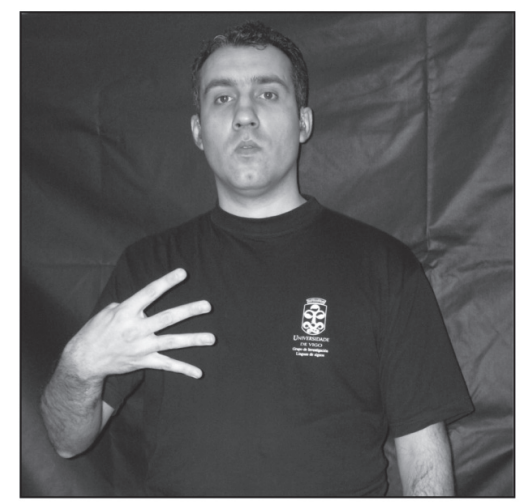

Figura 4. CUARTO_PUESTO (en una clasificación) 
En la figura 4 nos referimos a alguien o algo que ocupa el cuarto lugar en una clasificación. En el vídeo del que tomamos este ejemplo el signante indicaba que si el equipo ganaba esa semana pasaría a la segunda posición en la tabla clasificatoria. Para expresar esta información se vale del espacio, de manera que simultáneamente desplaza el signo hacia arriba (para expresar icónicamente su subida) y la mano adopta la configuración V («2»)

Esta expresión es la utilizada también cuando indicamos en qué piso vivimos; puesto que la visión estándar de un edificio es por plantas horizontales, la mano adopta esa orientación y reproduce una dirección horizontal. Sin embargo, si transmitimos una información relacionada con la puerta en la que vivimos, de un pasillo o un patio de casas, los dedos de la mano se orientan hacia arriba.

De manera similar a la vista en la figura 4, la siguiente es otro ejemplo de cómo podemos utilizar el espacio para expresar una información numérica y de orden. El signante habla de las diferentes fases de clasificación de un campeonato (en este caso, la liga de campeones de la UEFA). La primera imagen hace referencia a las fases anteriores a los cuartos de final y a continuación se desplazan los articuladores hacia arriba, expresando icónicamente el orden ascendente en el torneo, al tiempo que las manos van marcando en qué fase nos encontramos -cuartos de final, semifinales y final-, a través de configuraciones numéricas que reflejan, en cierta medida, los términos a los que hacen referencia: cuartos «4», semifinales «2», final «1». El último signo de la serie es icónico y se emplea sobre todo en el discurso espontáneo puesto que existe un signo estándar para referirse a una final (figura 6).

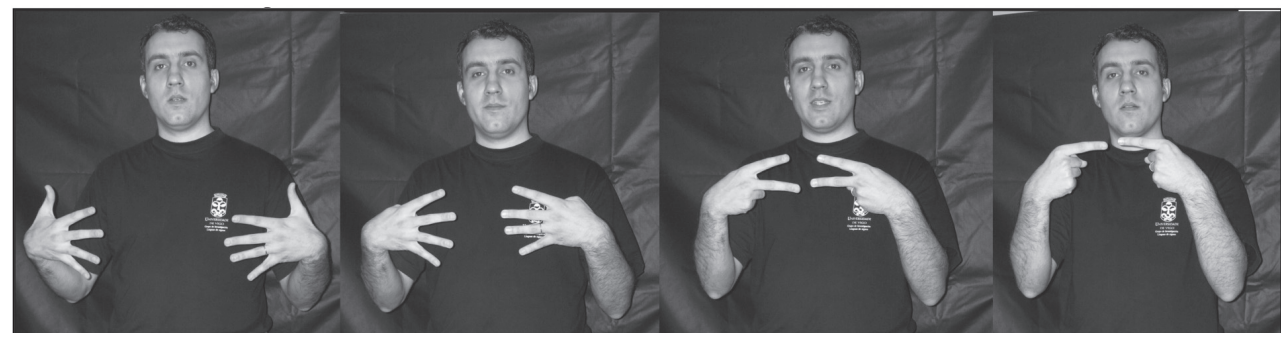

Figura 5. OCTAVOS CUARTOS SEMIFINALES FINAL

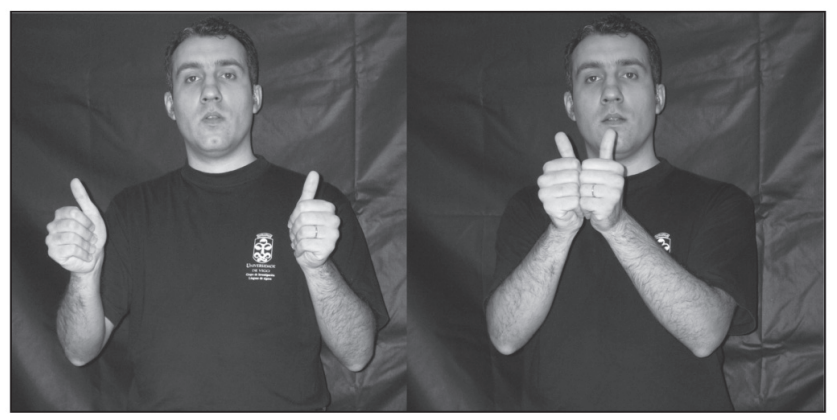

Figura 6. FINAL (signo estándar) 
La imagen siguiente muestra un signo que transmite un contenido numérico, relacionado también con el ámbito futbolístico, en un contexto discursivo. El signante habla del resultado de un partido de fútbol en el que el equipo de casa ha vencido por 4 a 1 . La localización de cada articulador en el espacio permite transmitir, gracias a la posición de cada mano, quién es el ganador y quien el vencedor y cuál ha sido el tanteo del partido. Este tipo de estructuras parece utilizarse solamente cuando el equipo ganador es el que juega en casa; si se diera el caso contrario se usarían signos estándar para explicar el resultado.

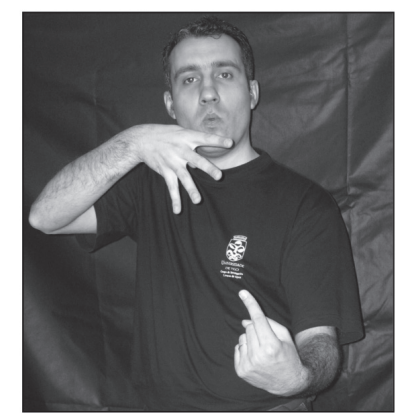

Figura 7. RESULTADO: 4-1

Liddell (2003: 182) observa en ASL casos en los que los signantes hablan de puntuaciones obtenidas en un concurso y hacen asociaciones entre los jugadores y los tanteos, de manera que el signo numeral que corresponde a la puntuación de cada uno se realiza en las posiciones del signante y de su interlocutor: «The placement of the hand producing the numeral sign associates the numerical value of the sign with the individual that the hand point toward». En ambas lenguas encontramos pues un recurso similar; se trata de contextos en los que la asociación de la información léxica aportada por los signos numerales y la localización espacial de los mismos nos permiten transmitir un significado complejo con una gran economía de realización.

(5) He dejado el coche aparcado en la planta menos cuatro del parking.

MI COCHE CL:COCHE_APARCAR CUARTA_PLANTA_SUBTERRÁNEA

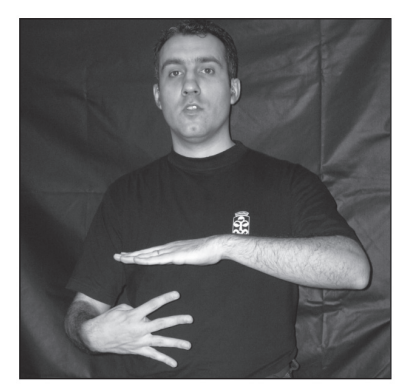

Figura 8. CUARTA_PLANTA_SUBTERRÁNEA 
En esta figura, la proforma de la mano dominante es idéntica a la de la figura 4 pero se desplaza en el espacio hacia abajo y se añade un nuevo articulador que representa la superficie que está encima de un aparcamiento subterráneo. En el caso anterior indicamos que con aquel signo podíamos hacer referencia al piso o planta en la que vivíamos. En este ejemplo, la localización del signo debajo de la mano pasiva-el suelo - aporta la lectura de que se trata de un piso bajo tierra.

(6) Ahí enfrente hay una hilera de coches. El mío es el cuarto por la derecha.

COCHE ENFRENTE [BIM]CL:COCHE[N: MULT][LOC X,Y,Z...] // MI COCHE CL:COCHE[N: MULT][LOC W, X,Y] CUARTO_POR_LA_DERECHA[LOC Z]

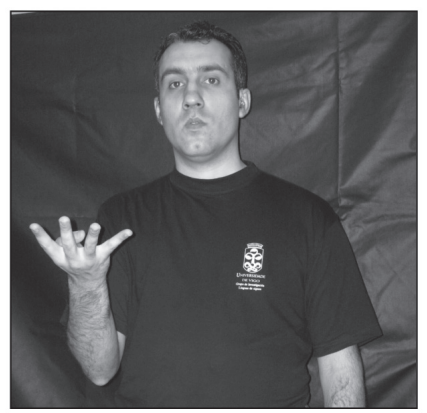

Figura 9. CUARTO_POR_LA_DERECHA

El signante se está refiriendo al lugar que ocupa su coche en una fila de ellos. Primero describe la situación; luego articula la proforma referida a la forma de los coches, solo con la mano derecha (con un movimiento de derecha a izquierda) en el espacio tres veces y a continuación coloca su coche en la cuarta posición empezando por la derecha, empleando el signo que aparece en la figura 9. La orientación de la mano refleja la visión horizontal de una hilera de coches.

(7) Las entradas para la película están en la cuarta fila.

ENTRADA CINE FILA[N: MULT][LOC X, Y, Z] CUARTA_FILA

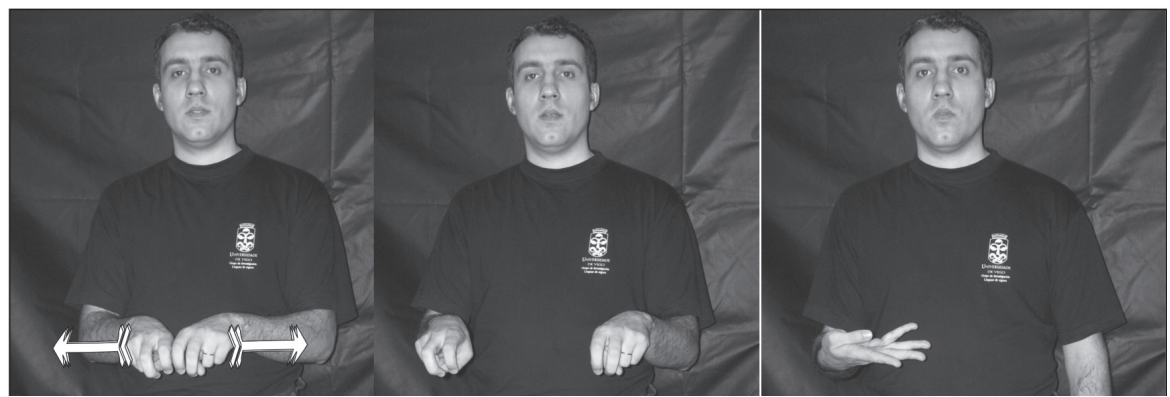

Figura 10. FILA CUARTA_FILA 
Esta imagen transmite también una información de orden y localización unida a la visión de un patio de butacas. En este caso la orientación de la palma es la misma que en el ejemplo anterior, pero la orientación de los dedos reproduce la perspectiva horizontal de las filas de un cine; el signo se realiza hacia el cuerpo del signante, tomando el espacio sígnico como el lugar de la pantalla y el cuerpo como el límite posterior de la sala de proyección.

Al lado de estos ejemplos encontramos otros que están menos estandarizados en la lengua y que son, por lo tanto, más discursivos. En el primero de ellos (figura 11) el espacio adquiere un triple valor de marcador de orden, localización e igualdad referencial. La mano actúa como mapa referencial.

(8) Los cinco números del premio gordo de la lotería coinciden con mi boleto.

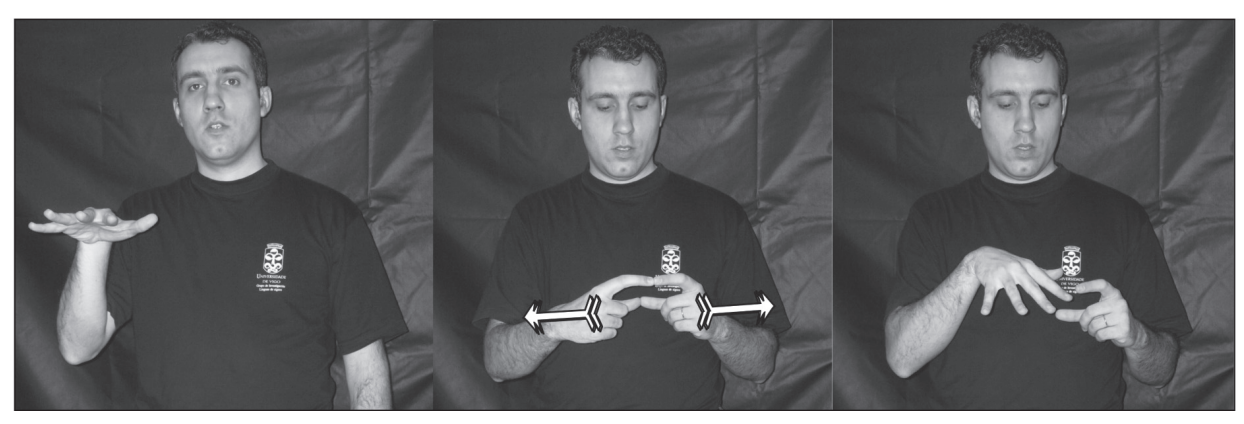

Figura 11. CINCO_NÚMEROS[LOC X] DÉCIMO[LOC Y] [MNODOM]DÉCIMO[LOC Y]/ CINCO_NÚMEROS[LOC Y]

La localización del primer signo está motivada; el signante lo orienta hacia el lugar en el que se sobreentiende que está la televisión en la que está viendo el sorteo de lotería ${ }^{11}$. De allí hace una transferencia hacia su mano pasiva que mantiene parte de la articulación del signo DÉCIMO para marcar la igualdad referencial de los referentes: los números del premio y su décimo de lotería. Los elementos de la narración se hacen presentes en el espacio de la enunciación, se ubican en él como si estuvieran presentes.

(9) Otras veces había tenido la terminación o incluso las tres últimas cifras.

EN-EL-PASADO PREMIO Q $\overline{\mathrm{UE} / /}$ qu

NÚMERO_TERMINACIÓN[LOC X] O TRES_ÚLTIMAS_CIFRAS[LOC X]

11 Es un ejemplo tomado del texto que se muestra en el apartado 1: "Las navidades pasadas me tocó la lotería. Cuando vi en la tele que los cinco números coincidían con mi boleto, no me lo podía creer. Otras veces había tenido la terminación e incluso una vez las tres últimas cifras, pero nunca el premio gordo". 


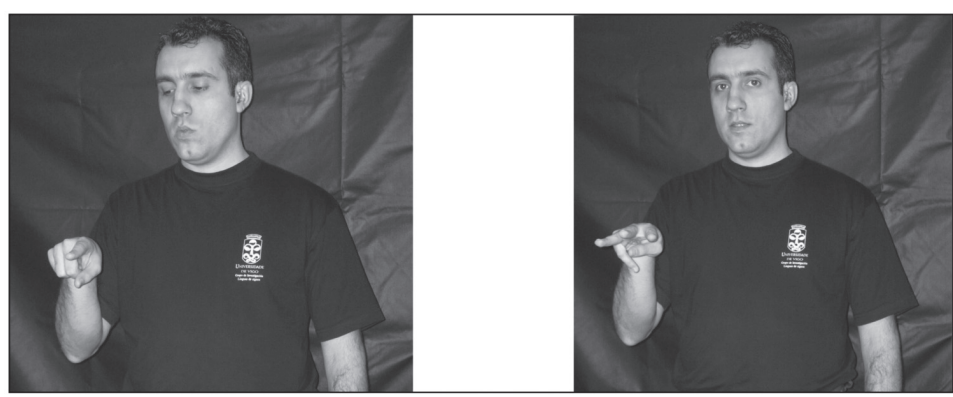

Figura 12. NÚMERO_TERMINACIÓN[LOC X]

TRES_ÚLTIMAS_CIFRAS[LOC X]

La mirada adquiere también en este caso un papel fundamental. El signante la dirige hacia el objeto protagonista de la enunciación, hecho que activa los puntos espaciales en los que articula los diferentes signos. En este ejemplo la localización de los signos referidos a las terminaciones coincide con la localización de CINCO_NÚMEROS (figura 11), aunque el signante se refiere a dos momentos temporales diferentes; esto implica que el signante asume un mismo contexto de actuación en todas las ocasiones: comprueba los números de la lotería en la televisión.

\subsubsection{La explotación del espacio sígnico en contextos miméticos}

Se trata de contextos en los que el signante reproduce, de manera idéntica a la realidad, la realización de una acción. En este tipo de acciones (representadas) hacemos uso de la iconicidad metafórica. En palabras de Herrero Blanco (2009: 380):

La iconicidad metafórica se produce cuando los signos o palabras se usan en lugar de los objetos reales mismos, y el contexto de la signación se toma como contexto real. El signante se ubica en ese contexto, y las referencias en él resultan análogas a las del contexto real.

En el ejemplo siguiente podemos observar estas características. El signante representa la acción relativa a la manera de colocar la palanca de cambios de un coche en diferentes posiciones (marchas). La articulación de estos signos informa, simultáneamente, sobre la manera cómo se realiza la acción de meter las marchas y sobre cuál estamos accionando; representa, exactamente, su realización en un contexto real.

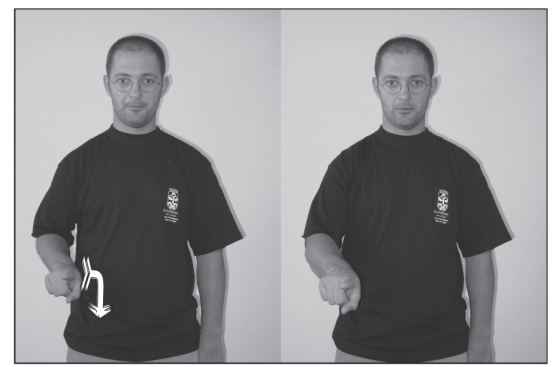

Figura 13. PRIMERA 


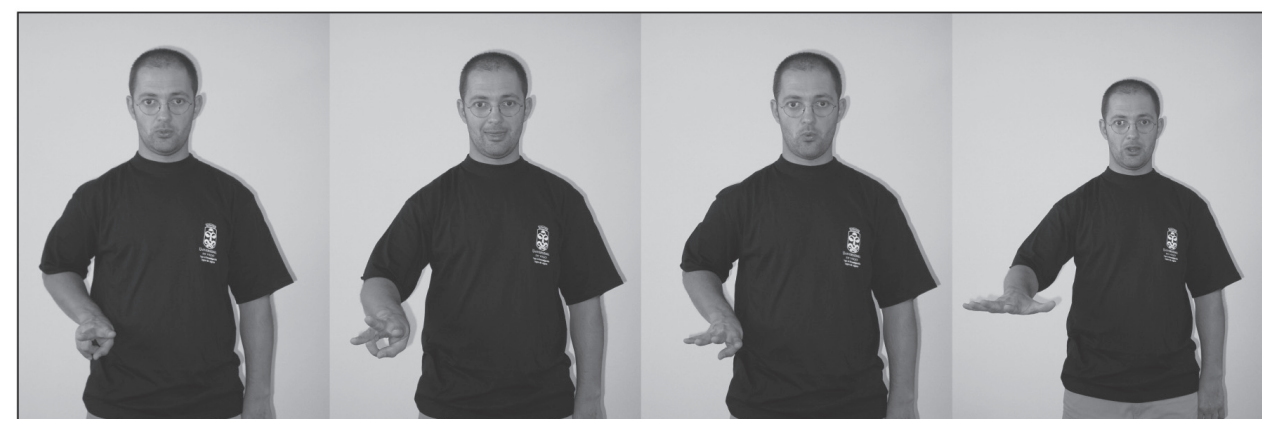

Figura 14. SEGUNDA TERCERA CUARTA QUINTA

En la expresión de esta información, observamos además como el signante recurre a otro procedimiento propio de las lenguas de signos: la incorporación numeral. La articulación de los signos reproduce el movimiento que realizamos para cambiar de marcha en un coche y además del movimiento, la proforma empleada varía aportando la información numérica asociada a cada marcha.

\section{Reflexiones finales}

La explotación del espacio sígnico que caracteriza a las lenguas de signos es un recurso rico y económico que permite la expresión de un gran número de conceptos. Esta explotación nos ofrece la posibilidad no solo de situar los signos en el espacio sino también de usar dicho espacio de manera simbólica para representar diferentes situaciones. Esta representación puede presentar un alto grado de iconicidad si recurrimos a características espaciales reconocibles a la hora de describir una situación determinada. Es una característica que las lenguas de signos emplean de manera recurrente.

En este trabajo nos hemos acercado al estudio de los numerales en contextos en los que su articulación base sufre algunas modificaciones para expresar referencias temporales, espaciales... con un alto grado de iconicidad.

En los contextos analizados la iconicidad está asociada a la construcción, en el espacio, tanto de referencias temporales, tomando el espacio como mapa temporal, como de referencias espaciales, para expresar informaciones numéricas referidas a entidades localizadas en un espacio real que se reproduce en un espacio sígnico simbólico.

Hemos analizado, en primer lugar, la utilización del espacio sígnico como mapa temporal y la expresión de información numérica unida a referencias temporales ${ }^{13}$. Al lado de la expresión básica de los cuantificadores numerales y de los procesos de incorporación numeral con los signos que expresan tiempo, existen en la LSE otros procedimientos que permiten crear puntos de referencia en el espacio a través de la interpretación de este como un calendario; en ocasiones, los puntos de referencia se crean en la mano pasiva, cuando a la información temporal se le añade una información de orden, referida en todos los casos analizados a las semanas del mes.

13 Cabeza y Fernández (2004) presentan un estudio exhaustivo de la expresión del tiempo en LSE. 
En nuestra opinión estos son procedimientos que se engloban dentro de la iconicidad diagramática: una secuencia temporal se interpreta en el espacio sígnico concebido como un diagrama en el que se construye una secuencia temporal.

En segundo lugar hemos abordado la explotación del espacio sígnico para expresar información numérica asociada a entidades que se localizan en un determinado punto del espacio que actúa en este caso como un mapa a escala del mundo real. Los signos transmiten no solo una información locativa sino que su posición en el espacio nos permite también extraer una información de orden e incluso de igualdad referencial entre entidades localizadas en diferentes puntos del espacio. En estos contextos la construcción de referencias en el espacio se apoya en la iconicidad imagética.

Por último hemos observado aquellos contextos en los que el espacio sígnico se convierte en un espacio real en el que se reproduce una acción de manera análoga a la realidad, nos encontramos en la dimensión de la iconicidad metafórica.

Este análisis nos ha permitido corroborar dos importantes premisas; por una parte, hemos confirmado la idea de que las lenguas de signos, y en concreto la LSE, recurren no solo al espacio lingüístico para la realización de sus signos sino también a un espacio topográfico que permite la representación a escala del mundo real en el espacio de signación; por otra parte, hemos certificado que la utilización del espacio en la LSE no puede separarse de la noción de iconicidad.

La explotación del espacio no solo con fines lingüísticos sino también con fines discursivos nos ha permitido analizar los tres niveles de iconicidad que podemos encontrar en las lenguas: imagética, diagramática y metafórica y nos ha permitido ahondar en las posibilidades discursivas que presenta la LSE. En nuestra opinión, el estudio de las características lingüísticas de las lenguas de signos no debe alejarse del estudio de la iconicidad como elemento descriptivo para poder analizar cómo se expresa la información que se aleja de la norma gramatical y posee por ello un carácter más discursivo.

\section{Referencias bibliográficas}

Armstrong, David F. (1983): «Iconicity, arbitrariness, \& duality of patterning in signed and spoken languages. perspectives on language evolution», Sign Language Studies, 38, págs. 51-69.

Cabeza Pereiro, C. y A. Fernández Soneira (2004): «The Expression of Time in LSE». Sign Language \& Linguistics, 7:1, Amsterdam/Philadelphia, John Benjamins, págs. 63-82.

Cuxac, C. (1996): Fonctions et structures de l'iconicité des langues des signes. Thèse de Doctorat d'Etat, Université Paris V.

Cuxac, C. (2000): La Langue des Signes Française (LSF). Les voies de l'iconicité. Paris, Ophrys.

Cuxac, C. (2003): «Iconicité des langues des signes: mode d'emploi». Cahiers de linguistique analogique, 1, ABELL (Association Bourguignonne d'Etudes Linguistiques et Littéraires), págs. 239-264.

Emmorey, K. (2002): Language, cognition and the brain: Insights from sign language research. Hillsdale, NJ, Lawrence Erlbaum Associates.

Fernández Soneira, A. (2008): La cantidad a manos llenas. La expresión de la cuantificación en la lengua de signos española. Madrid, Fundación CNSE.

Frishberg, Nancy (1975): «Arbitrariness and iconicity: historical change in American Sign Language». Language 51, págs. 696-719. 
García-Miguel Gallego, J. Ma (1996): «O princípio de abitrariedade e a lingüística actual». En Henríquez, M. C. (ed.): Actas do IV Congresso Internacional da Língua Galego-Portuguesa na Galiza en Homenagem a Ferdinand de Saussure. Orense, Associaçom Galega da Língua, págs. 415-422.

Gascón. A. (1998): «¿Señas o signos?: evolución histórica». Comunicación presentada en el I Congreso ibero-americano de educación bilingüe para sordos, Lisboa: http://www.ucm.es/info/civil/bardecom/docs/signos.pdf (03/09/2009).

Haiman, J. (1980): «The iconicity of grammar». Language, 56/3, págs. 515-540.

Haiman, J. (ed.) (1985): Iconicity in Syntax. Amsterdam/Philadelphia, John Benjamins.

Herrero Blanco, Á. (2009): Gramática didáctica de la lengua de signos española (LSE). Madrid, Ediciones SM / Fundación CNSE.

Jakobson, R. (1971): «Quest for the essence of language». En R. Jakobson: Select Writings II. Word and Language, The Hague - Paris, Mouton, págs. 345-359 [Traducción del original en francés «A la recherche de l'essence du langage» publicado en Diogenes 51, págs. 22-38].

Johnston, T. (1996): A.U.S.L.A.N Dictionary. A Dictionary of the Sign Language of the Australian Deaf Community. Australia, Deafness Resources Australia.

Klima, Edward y Ursula Bellugi (1979): The signs of language. Cambridge, Mass., Harvard University Press.

Liddell, S. K. (1990): «Four Functions of a Locus: Reexamining the Structure of Space in ASL». En Lucas, C. (ed.): Sign Language Research. Washington D.C., Gallaudet University Press, págs. 176-198.

Liddell, S. K. (1995): «Real, surrogate and token space: Grammatical consequences in ASL». En Emmorey, K \& J. Reilly (eds.): Language, gesture and space. Mahwah, NJ, Lawrence Erlbaum Associates, págs. 19-41.

Liddell, S. K. (2003): Grammar, Gesture and Meaning in American Sign Language. Cambridge, Cambridge University Press.

Peirce, C. S. (1987): «El icono, el índice y el símbolo». En Peirce, C. S.: Obra lógico-semiótica. Madrid, Taurus, págs. 261-303. [Trad. del capítulo correspondiente a los parágrafos 2274-2778 de los Collected Papers of Charles Sanders Peirce, ed. The Belknap Press of Harvard University Press, Cambridge, Massachusetts, 1965].

Talmy, L. (2006): «The representation of spatial structure in spoken and signed language». En Hickmann, M. \& S. Robert (eds.): Space in Languages. Linguistic Systems and Cognitive Categories. Amsterdam/Philadelphia, John Benjamins, págs. 207-238.

Taub, S. (2001): Language from the body: iconicity and metaphor in American Sign Language. Cambridge, Cambridge University Press.

Sallandre, M. A. (2003): Les unités du discours en Langue des Signes Française. Tentative de catégorisation dans le cadre d'une grammaire de l'iconicité. Thèse de Doctorat d'Etat, Université de Paris VIII.: http://umr7023.free.fr/Downloads/Sallandre these_tabmat.html (05-11-2007).

Sallandre, M. A. (2006): «Iconicity and space in French Sign Language». En M. Hickmann \& S. Robert (eds.): Space in Languages. Linguistic Systems and Cognitive Categories. Amsterdam/Philadelphia, John Benjamins, págs. 239-255.

Simone, Raffaele (ed.) (1995): Iconicity in language. Amsterdam/Philadelphia, John Benjamins.

Wilcox, P. (2000): Metaphor in American Sign Language. Washington D.C., Gallaudet University Press. 